\title{
Micro-scale Cellular Automaton Modeling of Interface Evolution During Reaustenitization from Pearlite Structure in Steels
}

\author{
Gang Shen ${ }^{1} \cdot$ Cheng-Wu Zheng ${ }^{2} \cdot$ Jian-Feng Gu ${ }^{1,3,4} \cdot$ Dian-Zhong $\mathrm{Li}^{2}$ \\ Received: 1 September 2017 / Revised: 9 November 2017/Published online: 6 February 2018 \\ (C) The Chinese Society for Metals and Springer-Verlag GmbH Germany, part of Springer Nature 2018
}

\begin{abstract}
A modified cellular automaton model is developed to depict the interface evolution inside the cementite plus ferrite lamellar microstructures during the reaustenitization of a pearlite steel. In this model, migrations of both the austeniteferrite and austenite-cementite interfaces coupled with the carbon diffusion and redistribution are integrated. The capillarity effect derived from local interface curvatures is also carefully considered by involving the concentration given by the phase diagram modified by the Gibbs-Thomson effect. This allows the interface evolution from a transient state to a steady state under different annealing conditions and various interlamellar spacings to be simulated. The proposed cellular automaton approach could be readily used to describe the kinetics of austenite formation from the lamellar pearlites and virtually reveal the kinematics of the moving interfaces from the microstructural aspect.
\end{abstract}

Keywords Austenite formation - Lamellar pearlite $\cdot$ Interface evolution $\cdot$ Cellular automaton $\cdot$ Microstructural modeling

\section{Introduction}

Solid-state phase transformation is one of the most attractive metallurgical phenomena in manufacturing metallic materials, especially steels, in order to tailor or optimize the material properties [1-3]. Among the phase transformations in steels, austenite $(\gamma)$ formation from matrix consisting of ferrite $(\alpha)$ and cementite $(\theta)$ aggregates by reheating to partially or fully austenitic region is gaining

Available online at http://link.springer.com/journal/40195

Cheng-Wu Zheng

cwzheng@imr.ac.cn

$\triangle$ Jian-Feng Gu gujf@sjtu.edu.cn

1 Institute of Materials Modification and Modelling, School of Materials Science and Engineering, Shanghai Jiao Tong University, Shanghai 200240, China

2 Shenyang National Laboratory for Materials Science, Institute of Metal Research, Chinese Academy of Sciences, Shenyang 110016, China

3 Materials Genome Initiative Center, Shanghai Jiao Tong University, Shanghai 200240, China

4 Shanghai Key Laboratory of Materials Laser Processing and Modification, Shanghai Jiao Tong University, Shanghai 200240, China more interest due to the increasing demand in microstructure refinement and property improvement in steel production such as dual-phase steel [4] and transformationinduced plasticity steel [5]. Austenite conditions, e.g., composition and morphology, at high temperatures can strongly affect the final microstructure and mechanical properties of steel products. Many experimental efforts have been made to understand the metallurgical theory, transformation kinetics and microstructural morphology during austenite formation from steels with different compositions and initial microstructures [6-9].

For steels in pearlitic condition, the initial microstructure is made up of an array of pearlite colonies, with each consisting of an interpenetrating bicrystal of ferrite and cementite, which has an appearance of alternate thin layers of ferrite and cementite in a two-dimensional view [10,11]. Due to the complexity in spatial arrangement of the microstructural constitutions, austenite formation from pearlite structures is a more sophisticated process compared with spheroidized ferrite-cementite microstructures. Anisotropic growth of newly formed austenite along and vertical to lamella direction of a certain pearlite colony is observed by Cerda et al. [12] during continuous heating of low- and medium-carbon steels. Maximum growth rate is reached at the triple junctions where austenite, ferrite and cementite phases meet due to the sufficient carbon 
available from cementite and minimum diffusion distance for carbon to feed to the growing front. To quantitatively describe the microstructure evolution kinetics during reaustenitization process, several numerical models have been proposed based on analytical solutions to diffusioncontrolled growth equations [13-15] or phenomenological approaches like the Johnson-Mehl-Avrami-Kolmogorov theory [16]. However, these models cannot provide any direct view inside the microstructure evolution process during reautenitization.

With the advancement of computational capabilities of modern computers and also the abundant numerical algorithms available for solving partial differential equations, it is now possible to model microstructure evolution process at microscale, i.e., at the length scale of individual microstructure features using various microscopic approaches, such as cellular automaton (CA), Monte Carlo (Potts) and phase field method [17]. Because of the intrinsic flexibility in definition of cell states and transition rules, CA method is more suitable for modeling the complexity of microstructure morphology and phase transformation kinetics [18]. Some sophisticated CA models have already been proposed to simulate the microstructure evolution during austenite formation from either hypoeutectoid or eutectoid steels [19-21]. Yang et al. [19] and Zhu et al. [21] developed CA models considering different nucleation and growth behaviors within and along the edges of the pearlite colonies to simulate the microstructure evolution in terms of morphology and transformation kinetics during reaustenitization. However, due to the considerably large dimensional difference between the thickness of a single cementite lamella and the size of the pearlite colony, it is usually impossible to model a microstructure considering large numbers of grain structures and the pearlite lamellar geometry at the same time. Pearlite structures in these models are simplified as an effective phase with uniform carbon concentration [21-23]. Definitely, local growing features and interface migration kinematics of austenite formation from pearlite structures can only be revealed when the detailed lamellar structure is carefully treated and modeled.

In this work, a modified CA model is developed to depict the evolution of interface migration inside the cementite plus ferrite lamellar microstructures. In this CA model, migrations of the $\gamma / \alpha$ and $\gamma / \theta$ interfaces coupled with the diffusion and redistribution of carbon are carefully integrated. The Gibbs-Thomson effect derived from local interface curvatures is also considered to derive a more reasonable interface shape at the growing front of austenite. The kinematics and morphologies of the austenite moving interface are then simulated under different annealing conditions using the proposed CA method.

\section{Model Descriptions}

\subsection{Interface Migration of Austenite}

During the dissolution of pearlitic lamellae, two different situations of the moving interface are usually considered. One is related to the dissolution of cementite due to austenite formation within the pearlite. The other is about continuous growing of the newly formed austenite at the expense of the ferrite. During this process, austenite growth is mainly limited by carbon diffusion inside the austenite, as schematically represented in Fig. 1. In order to describe the two situations of austenite interface migration, a mixedmode growth kinetics model [20] is adopted. The interface velocity can be described by:

$v_{i j}=M^{i j} \Delta G$,

where $v_{i j}$ is the interface moving velocity, $M^{i j}$ is the interface mobility, and $\Delta G$ is the chemical driving force. The indicators $i$ and $j$ represent different phases across the moving interfaces. The interface mobility can be calculated by:

$M^{i j}=M_{0}^{i j} \exp \left(-Q_{i j} /(R T)\right)$,

where $M_{0}^{i j}$ is a pre-exponential factor, $Q_{i j}$ is the activation energy for boundary migration, and $R$ and $T$ are the universal gas constant and the absolute temperature, respectively.

The chemical driving force $\Delta G$ is considered to be in first approximation as proportional to the derivation from the equilibrium concentration on the moving interface. For the $\gamma / \theta$ interface, the driving force for austenite growing toward cementite can be rewritten as:

$\Delta G_{\gamma \theta}=\chi_{(T)}^{\gamma \theta}\left(c_{\gamma / \theta}^{\mathrm{eq}}-c_{\gamma / \theta}\right)$,

where $\chi_{(T)}^{\gamma \theta}$ is a proportional factor, which can be determined using thermodynamic software, e.g., Thermal-Calc; $c_{\gamma / \theta}^{\mathrm{eq}}$ is carbon concentration of austenite in equilibrium with cementite, and $c_{\gamma / \theta}$ is carbon concentration of austenite at the moving $\gamma / \theta$ interface. As for the $\gamma / \alpha$ interface, the driving force for austenite growing toward ferrite can be described by:

$\Delta G_{\gamma \alpha}=\chi_{(T)}^{\gamma \alpha}\left(c_{\gamma / \alpha}-c_{\gamma / \alpha}^{\mathrm{eq}}\right)$,

where $\chi_{(T)}^{\gamma \alpha}$ is the proportional factor, $c_{\gamma / \alpha}$ is the carbon concentration of austenite at the moving $\gamma / \alpha$ interface, and $c_{\gamma / \alpha}^{\mathrm{eq}}$ is the carbon concentration of austenite in equilibrium with ferrite. 

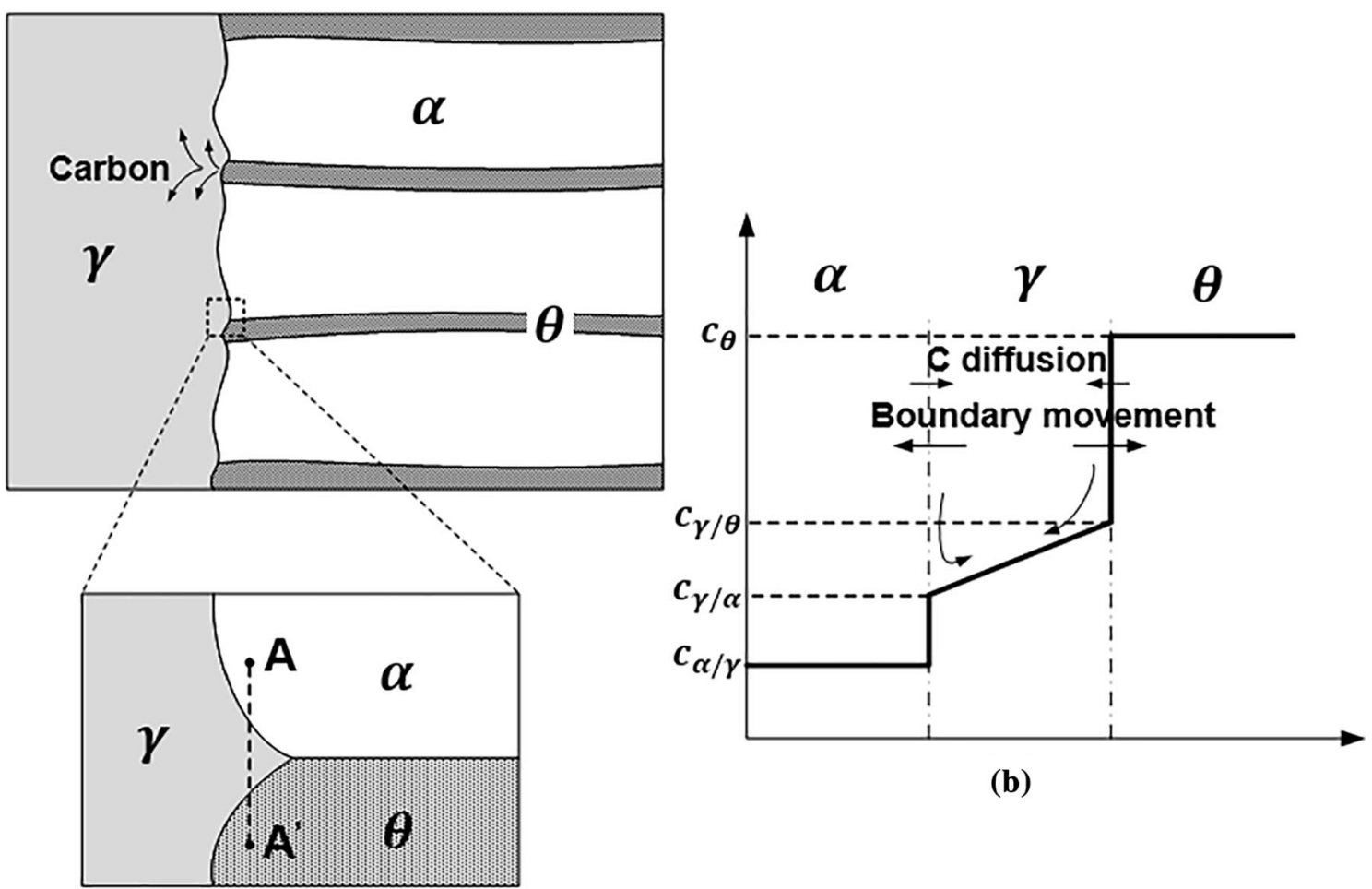

(b)

(a)

Fig. 1 Schematic representation of microstructure during dissolution of pearlite into austenite a and variation of carbon content along the dashed line $\mathrm{A}-\mathrm{A}^{\prime} \mathbf{b}$

\subsection{Gibbs-Thomson Effect}

Capillarity does influence the migration kinetics and morphology of the interface during dissolution of the pearlite structures. Here, the effect of local curvature is taken into account via modifying the thermodynamic equilibrium as

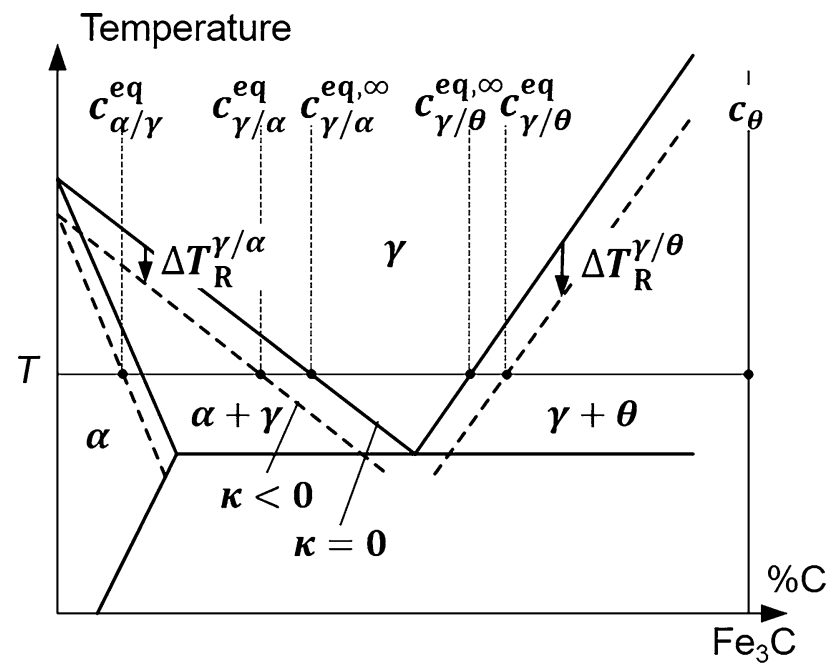

Fig. 2 Schematic representation of linearized $\mathrm{Fe}-\mathrm{C}$ phase diagram with Gibbs-Thomson effect and definition of concentrations imposed at interface for CA calculation in Ref. [24]. As shown in Fig. 2, the phase diagram is modified for the local curvature via the Gibbs-Thomson coefficient. The interfacial concentrations for given local curvature at temperature $T$ can be determined as [25]:

$c_{\gamma / \alpha}^{\mathrm{eq}}(T, \kappa)=c_{\gamma / \alpha}^{\mathrm{eq}, \infty}\left(T-\Gamma_{\gamma / \alpha} \kappa\right)$,

$c_{\gamma / \theta}^{\mathrm{eq}}(T, \kappa)=c_{\gamma / \theta}^{\mathrm{eq}, \infty}\left(T-\Gamma_{\gamma / \theta} \kappa\right)$,

where $c_{\gamma / \alpha}^{\mathrm{eq}, \infty}(T)$ and $c_{\gamma / \theta}^{\mathrm{eq}, \infty}(T)$ are the concentrations given by the phase diagram for a planar interface at temperature $T$. In this way, change in the equilibrium carbon concentration due to the local curvature at the interface is compensated by introducing an overheating $\Delta T_{\mathrm{R}}=\Gamma \kappa$ given by the local curvature $(\kappa)$ and the Gibbs-Thomson coefficient $(\Gamma)$, which further influences the migration velocity of the interface as described in Eqs. (1-4). Negative curvature for a concave interface shape of austenite will reduce the equilibrium carbon concentration at $\gamma / \alpha$ interface $\left(c_{\gamma / \alpha}^{\text {eq }}\right)$ and increase the equilibrium carbon concentration at $\gamma / \theta$ interface $\left(c_{\gamma / \theta}^{\mathrm{eq}}\right)$ as described by the dashed line in Fig. 2.

Here, the local curvature is calculated using an approach known from the curvature calculation in the lattice-based model [26]: 
$\kappa=\frac{A}{C_{\text {cell }}} \frac{\text { Kink }-N_{i}}{N+1}$,

where $C_{\text {cell }}$ is the grid spacing in the cellular automaton model, $A=1.28$ is a topological coefficient, $N=24$ is the number of the first and second nearest neighbors for a square lattice, $N_{i}$ is the number of cells within the neighborhood belonging to grain $i$, and Kink $=14$ is the number of cells within the neighborhood belonging to grain $i$ for a flat interface $(\kappa=0)$. The topological considerations behind this model can be found in Ref. [27].

\subsection{Carbon Diffusion}

The growth kinematics of the newly formed austenite, including growth rate and interface morphology, is controlled by carbon diffusion from the decomposing cementite to $\gamma / \alpha$ interface $[14,28]$. Due to the low carbon solubility in ferrite, carbon diffusion in ferrite is neglected here. Only carbon diffusion in austenite is considered. The carbon diffusion in austenite is described by:

$\frac{\partial c_{\gamma}}{\partial t}=\nabla \cdot\left(D_{\mathrm{c}}^{\gamma} \nabla c_{\gamma}\right)$

where $c_{\gamma}$ is the carbon concentration in austenite, $D_{\mathrm{c}}^{\gamma}$ is the carbon diffusion coefficient in austenite, and $t$ is the time. The diffusion coefficient $D_{\mathrm{c}}^{\gamma}$ is assumed to be thermally activated according to:

$D_{\mathrm{c}}^{\gamma}=D_{\mathrm{c}, 0}^{\gamma} \exp \left(-Q_{\mathrm{c}}^{\gamma} /(R T)\right)$,

where $D_{\mathrm{c}, 0}^{\gamma}$ is the pre-exponential constant and $Q_{\mathrm{c}}^{\gamma}$ is the activation energy for carbon diffusion.

\section{Cellular Automaton Model}

In this work, a deterministic two-dimensional CA model for phase transformation [20] is modified by incorporating the models above to simulate the austenite formation behaviors during isothermal annealing of lamellar pearlitic steels. In this CA model, the spatial system is discretized onto a 2D regular equally spaced square lattice. The neighbors of a cell are defined by Moore's neighboring rule, which considers the nearest and second nearest eight cells. To quantitatively describe the metallurgical phenomena occurred during phase transformation, five state variables are defined on each CA cell. They are: (1) the phase state variable, which indicates whether a cell belongs to ferrite, cementite, austenite phase or contains $\gamma / \alpha, \gamma / \theta$ interfaces; (2) the grain states variable, an integer ranging from 0 to 180 , which indicates the grain orientation of a cell; (3) the carbon concentration variable; (4) the austenite transformation fraction variable quantifying the austenite fraction in a cell; and (5) the cementite fraction variable quantifying the cementite fraction in a cell. Values of state variables on each CA cell are determined by the respective previous states and the previous states of the respective neighbors according to the sub-models described in Sect. 2. The automaton performs by synchronously updating the state variables of all lattice cells in each time step.

A deterministic transformation rule is applied to determine phase state transition for each CA cell. At time $t^{i}$, the growth length $\Delta l^{\gamma / \phi}$ of an austenite cell toward one of its neighboring interface cells can be described as:

$\Delta l^{\gamma / \phi}=\int_{t^{i}}^{t^{i+1}} v^{\gamma / \phi} \mathrm{d} t$,

where $v^{\gamma / \phi}$ is the moving velocity of a $\gamma / \phi$ interface cell, which is calculated using Eq. (1). At time $t^{i+1}$, the growth length of a $\gamma / \phi$ interface cell is:

$l_{t^{i+1}}^{\gamma / \phi}=l_{t^{i}}^{\gamma / \phi}+\sum \Delta l^{\gamma / \phi}$,

where $L_{\mathrm{CA}}$ is the defined grid spacing of the CA lattice. If $l_{t^{i+1}}^{\gamma / \phi} \geq L_{\mathrm{CA}}$, the $\gamma / \phi$ interface cell will change to a $\gamma$ phase cell.

Details regarding integrating the constitutive and kinetic laws outlined above into the CA approach are given in Refs. [17] and [29]. Here, only the items relevant to the current simulation are outlined in more detail:

1. To resolve the individual ferrite and cementite lamella in pearlite, very fine grid spacing $\left(L_{\mathrm{CA}}\right)$ set as $1 / 50$ of the thickness of a cementite lamella is used.

2. Nucleation of austenite is not considered in the current model. A layer of austenite cells is predefined in the initial microstructure with carbon concentration set as the equilibrium concentration with ferrite at the annealing temperature $T$.

3. Pearlite structure is simplified as periodic arrangements of alternative ferrite and cementite lamellae with the interlamellar spacing $\lambda$.

4. Carbon concentration in ferrite and cementite are assumed to be constant during the entire transformation. Only carbon diffusion in austenite is considered in the simulations.

5. Local equilibrium is assumed in the transformation. The equilibrium carbon concentration at the moving interface is derived from the linearized $\mathrm{Fe}-\mathrm{C}$ diagram modified with the Gibbs-Thomson effect.

6. An open source code Fipy [30] based on a standard finite volume (FV) approach is used to solve the partial differential diffusion equation, i.e., Eq. 8. Fipy is less sensitive to time step setting and thus more 
computationally efficient compared with the commonly used explicit finite difference (FD) method.

\section{Simulation Setting}

A plain carbon steel with near-eutectoid composition, Fe$0.8 \mathrm{C}$ (wt $\%$ ), is used to investigate the reaustenitization process from a lamellar pearlitic microstructure. To resolve the very fine cementite lamella, the grid spacing of the CA lattice domain is set to $1 / 50$ of the cementite lamella thickness which varies with the interlamellar spacing of pearlite. Pearlite structure is simplified as periodic arrangement of alternative ferrite and cementite lamellae with the interlamellar spacing $\lambda$. Thus, only one layer of ferrite and cementite lamellae is considered in the simulations based on the definition of periodic interface conditions. The width of the layered initial microstructure is set to be the lamellar spacing investigated. The cementiteferrite width ratio $\left(k^{\theta / \alpha}\right)$, which equals the cementite-ferrite volume ratio, is calculated by:

$k^{\theta / \alpha}=\frac{x_{\mathrm{C}}^{\mathrm{P}}-x_{\mathrm{C}}^{\alpha}}{x_{\mathrm{C}}^{\theta}-x_{\mathrm{C}}^{\mathrm{P}}}$

where $x_{\mathrm{C}}^{\mathrm{P}}, x_{\mathrm{C}}^{\theta}$ and $x_{\mathrm{C}}^{\alpha}$ are the mole fraction of carbon in the investigated steel, cementite and ferrite, respectively. The key parameters used in the simulations are listed in Table 1.

\section{Results and Discussion}

\subsection{Sidewise Growth of Austenite at the Ferrite- Cementite Interface}

To verify the proposed CA model, sidewise growth of the planar austenitic layer at the ferrite-cementite interface, as schematically shown in Fig. 3, is firstly simulated at an annealing temperature of $1073 \mathrm{~K}$. The initial thickness of the cementite lamella $\left(r_{0}\right)$ is $0.5 \mu \mathrm{m}$. Evolution of the

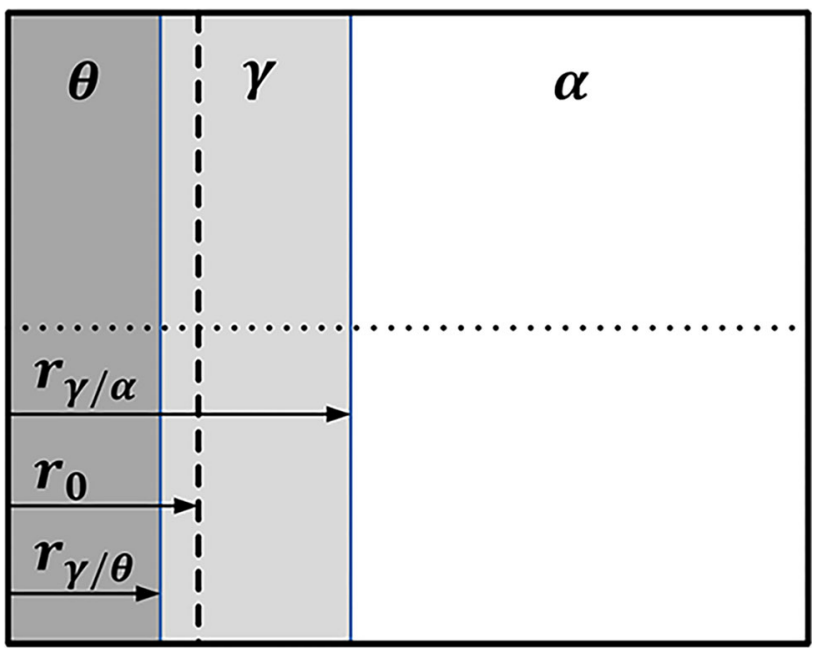

Fig. 3 Schematic representation of sidewise planar growth of austenite into ferrite and cementite $\left(r_{\gamma / \alpha}\right.$ and $r_{\gamma / \theta}$ are positions of the moving austenite fronts into ferrite and cementite, respectively)

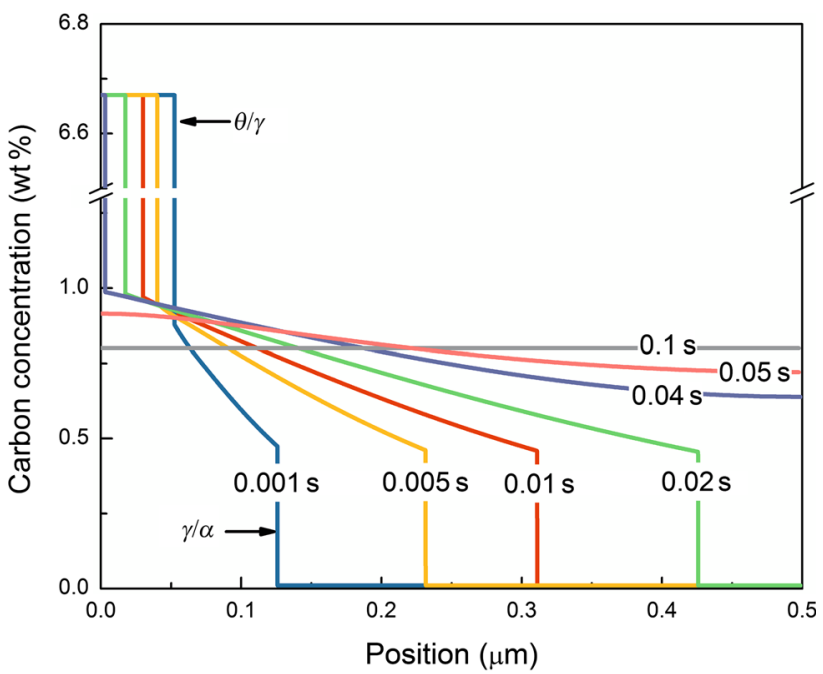

Fig. 4 Variation of carbon concentration along dotted line indicated in Fig. 3 at different time

carbon concentration profile along the growth direction obtained via CA is shown in Fig. 4. It can be seen that there exists a noticeable concentration gradient over the growing
Table 1 Key parameters used in simulations

\begin{tabular}{|c|c|c|c|}
\hline Symbol & Definition and unit & Value & Refs. \\
\hline $\mathrm{A}_{\mathrm{c} 1}$ & Austenite formation start temperature $(\mathrm{K})$ & 996.0 & {$[31]$} \\
\hline $\mathrm{A}_{\mathrm{c} 3}$ & Ferrite dissolution finish temperature $(\mathrm{K})$ & $-0.004021 \cdot T+4.7651$ & {$[31]$} \\
\hline$A_{\mathrm{ccm}}$ & Cementite dissolution finish temperature $(\mathrm{K})$ & $0.003255 \cdot T-2.4817$ & [31] \\
\hline$M^{\alpha \gamma}$ & Mobility of $\alpha / \gamma$ interface $\left(\mathrm{mol} \mathrm{m} \mathrm{J} \mathrm{J}^{-1} \mathrm{~s}^{-1}\right)$ & $0.5 \cdot \exp (-140000 /(R T))$ & {$[20]$} \\
\hline$M^{\gamma \theta}$ & Mobility of $\gamma / \theta$ interface $\left(\mathrm{mol} \mathrm{m} \mathrm{J} \mathrm{J}^{-1} \mathrm{~s}^{-1}\right)$ & $0.05 \cdot \exp (-140000 /(R T))$ & - \\
\hline$D_{\mathrm{c}}^{\gamma}$ & Carbon diffusion coefficient in austenite $\left(\mathrm{m}^{2} \mathrm{~s}^{-1}\right)$ & $1.5 \times 10^{-5} \exp (-142000 /(R T))$ & {$[20]$} \\
\hline$\Gamma_{\gamma / \alpha}$ & Gibbs-Thomson coefficient of $\alpha / \gamma$ interface ( $\mathrm{K} \mathrm{m}$ ) & $2 \times 10^{-7}$ & {$[25]$} \\
\hline$\Gamma_{\gamma / \theta}$ & Gibbs-Thomson coefficient of $\gamma / \theta$ interface $(\mathrm{K} \mathrm{m})$ & $2 \times 10^{-7}$ & {$[25]$} \\
\hline
\end{tabular}


austenite at the early stage of the transformation. Driven by this concentration gradient, carbon atoms gradually diffuse from the carbon-rich $\gamma / \theta$ interface into the austenite interiors to feed the newly formed austenite at the moving $\gamma / \alpha$ interface. Then, the austenite interface continuously moves into the ferrite concomitantly with gradual dissipation of cementite. During this process, carbon diffusion in austenite is the rate-controlling parameter. With the continuing growth of austenite, the increase in the carbon diffusion distance across the austenite gradually slows the growth rate of the austenite as seen in Fig. 5. With a purpose of comparison, growth of the austenite layer is also simulated using the DICTRA software proposed for diffusion-controlled phase transformation [32]. The calculated time for complete dissolution of ferrite and cementite is 0.029 and $0.042 \mathrm{~s}$ by CA and 0.028 and $0.049 \mathrm{~s}$ by DICTRA. Excellent agreement between these two models proves the validity of the CA model. The proposed CA model can provide excellent depictions of interface migration kinetics and carbon concentration distribution during austenite formation.

\subsection{Evolution of the Austenite Interface Inside the Pearlite Lamellae}

During the dissolution of pearlite, newly formed austenite also prefers to grow up along the $\alpha / \theta$ interface due to the surplus of carbon available in neighboring cementite lamella. The growth of austenitic phase into the lamellar structure of pearlite is also simulated. Here, initial pearlitic structure with an interlamellar spacing of $0.5 \mu \mathrm{m}$ containing a layer of alternate ferrite and cementite lamellae, as shown in Fig. 6, is used. Periodic boundary conditions are defined at the left and right sides of the calculation domain

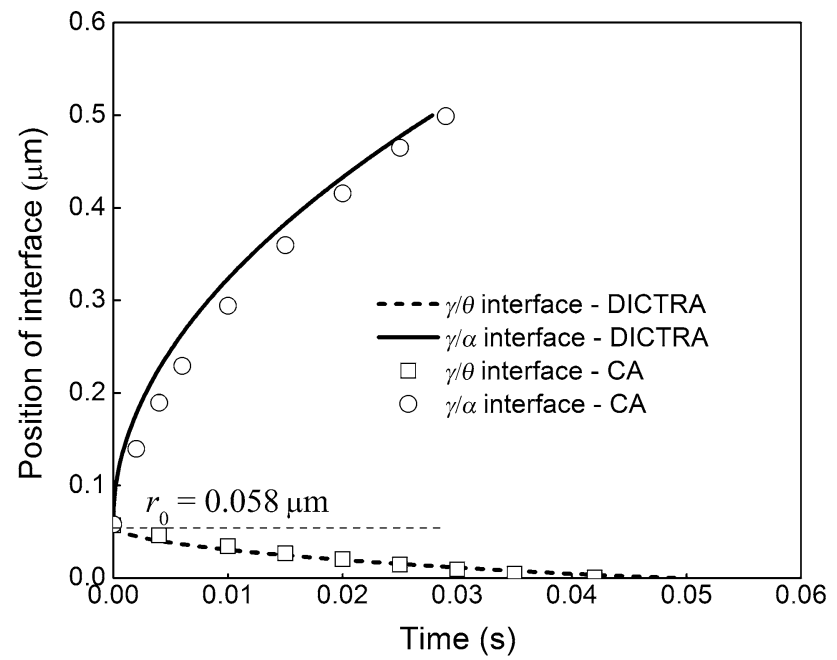

Fig. 5 Change in austenite interface positions predicted by CA and DICTRA

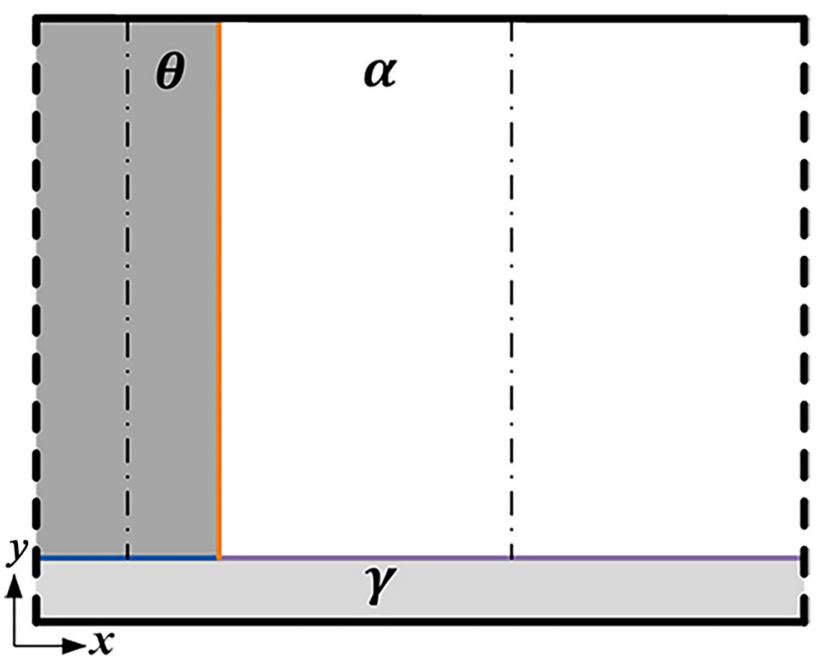

Fig. 6 Schematic representation of initial structure for austenite growing within pearlite lamella

(dashed lines in Fig. 6), which allows carbon diffusion across the boundaries. Adiabatic boundary conditions are defined at the upper and bottom sides of the calculation domain (black solid lines in Fig. 6), which prohibits carbon diffusion across the boundaries. A layer of austenite cells is predefined at the bottom of the calculation domain at the beginning of the calculation, and further growth of austenite performs by moving interface front cells without any nucleation process.

Simulation results of interface positions of the growing austenite during isothermal holding at $1073 \mathrm{~K}$ for different times are shown in Fig. 7a. It can be seen that at the beginning of the transformation, austenite grows predominantly along the $\alpha / \theta$ interface, where larger driving force for interface migration is available. With further processing of the transformation, the austenite growing front starts to bend with varied local curvatures introduced onto the migrating interface front. When the capillary effect caused by local curvature takes place, the transient migrating interface of the growing austenite gradually reaches a steady state, as shown in Fig. 7a, after which the interface of the growing austenite moves with a constant shape. The concentration field of carbon in austenite during the steady state is shown in Fig. 7b. It can be seen that the distribution of carbon concentration in the growing austenite is extremely inhomogeneous with carbon-rich and carbon-poor areas locating around the tip of the dissolving cementite and ferrite lamella, respectively.

Variation of local carbon concentrations along interface OD (as seen in Fig. 7) during the transformation is then traced as shown in Fig. 8. An obvious gradient of the carbon concentration does exist from the tri-junction where the $\alpha, \gamma$ and $\theta$ phases meet (i.e., the position O) toward the center of the $\gamma / \alpha$ interface (i.e., the position D). However, 


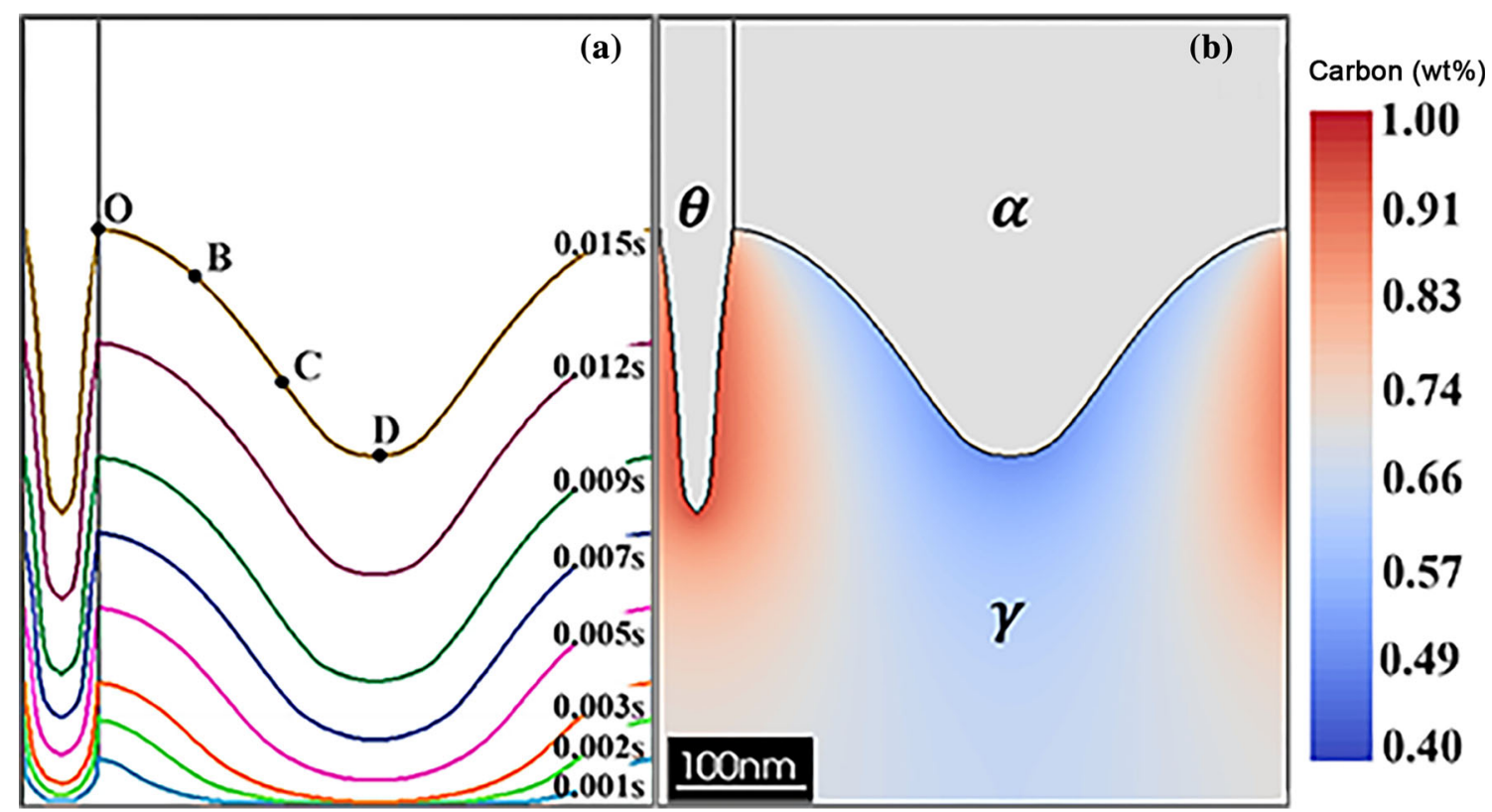

Fig. 7 Austenite growing inside pearlite lamella with interlamellar spacing of $0.5 \mu \mathrm{m}$ at $1073 \mathrm{~K}$ : a evolution of phase interface; b carbon concentration field at steady-state stage

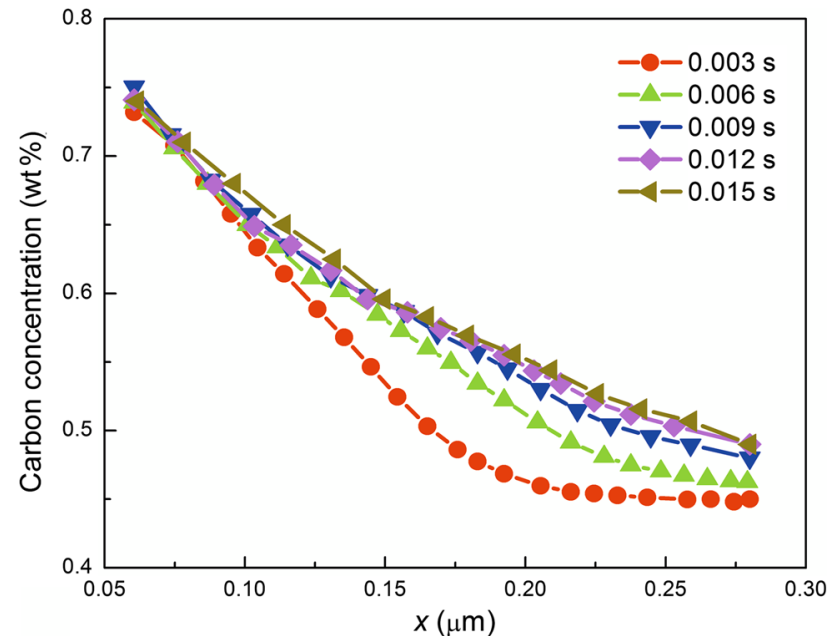

Fig. 8 Change in local carbon concentration profile along moving interface of OD as shown in Fig. 7

this carbon concentration gradient becomes nearly constant with further progressing of the transformation when the moving austenite interface approaches a steady migration state. The fact that carbon concentration gradient along the growing austenite interface is fairly constant can be found in the curvature contribution quantifying by the GibbsThomson effect: high positive curvature around the trijunction point lowers $c_{\mathrm{C}}^{\gamma / \theta}$ at the $\gamma / \theta$ interface and raises $c_{\mathrm{C}}^{\gamma / \alpha}$ at the $\gamma / \alpha$ interface, which reduces the concentration difference across the interface. However, the opposite occurs in regions with negative curvature far away from the tri-junction point.

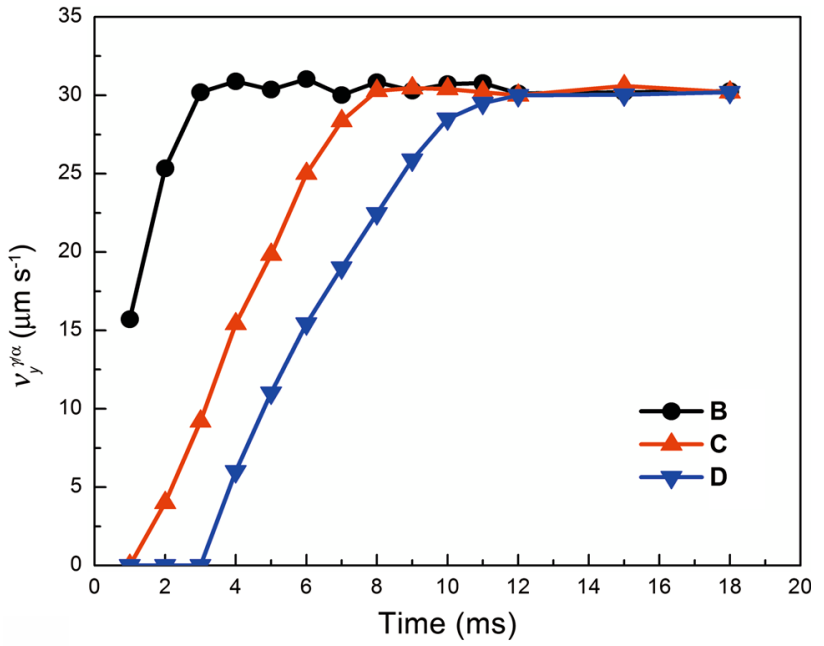

Fig. 9 Change in local velocities of the $\gamma / \alpha$ interface $\left(v_{y}^{\gamma / \alpha}\right)$ at different positions of $\mathrm{B}, \mathrm{C}$ and $\mathrm{D}$ (as shown in Fig. 7) of moving austenite interface

Figure 9 shows the changes in local migration velocities at different sites along the lamellar direction, which demonstrates transition of the interface migration mode from a transient state to steady state. It can be seen that the migration velocity of position $B$ near the tri-junction reaches its saturation quickly in less than $3.0 \mathrm{~ms}$. However, for the positions far away from $\alpha / \theta$ interface (i.e., position D), a comparatively long duration of initial transient is needed to reach the steady state. After which, the whole interface of austenite will migrate with a unity steady-state 
velocity and remain a fixed shape unless a certain fluctuation of carbon concentration breaks the balance.

\subsection{Effect of Carbon Diffusivity in Austenite}

In order to further examine the effect of carbon diffusivity in austenite on the interface migration kinematics, simulations with various carbon diffusion coefficients in austenite of $0.5 D_{\mathrm{C}}^{\gamma}, 1.0 D_{\mathrm{C}}^{\gamma}$ and $2.0 D_{\mathrm{C}}^{\gamma}$ are also performed. The simulation results of the interface shape and the carbon concentration profile in austenite are shown in Fig. 10. It can be seen that the carbon flux into austenite decreases with lower carbon diffusivity, resulting in the increase in concentration gradient in austenite, which causes a larger degree of bending of the wave-shaped migrating interface at steady state. Evolution of interface migration rate at the tip of dissolving ferrite lamella (position D in Fig. 7) with the above configuration of $D_{\mathrm{C}}^{\gamma}$ is exacted from CA simulation results and shown in Fig. 11. It is seen that $D_{\mathrm{C}}^{\gamma}$ affects not only the steady-state growth rate of austenite, but also the duration time of the transient growth period. As carbon diffusion coefficient alters from $0.5 D_{\mathrm{C}}^{\gamma}$ to 2.0 $D_{\mathrm{C}}^{\gamma}$, the steady-state growth rate of austenite increases from 23.6 to $38.0 \mu \mathrm{m} \mathrm{s}^{-1}$ for pearlite with interlamellar spacing of $0.5 \mu \mathrm{m}$ annealing at $1073 \mathrm{~K}$. Meanwhile, the duration time for transient growth period decreases from 14.0 to $7.0 \mathrm{~ms}$.

\subsection{Effect of Initial Interlamellar Spacing}

Figure 12 presents the results of the simulated steady-state interface shape together with the carbon concentration profile of austenite with three different interlamellar spacing of $0.2,0.4$ and $0.8 \mu \mathrm{m}$ at the soaking temperature of $1073 \mathrm{~K}$. The steady-state growth rates of the migrating interface with various interlamellar spacing are shown in

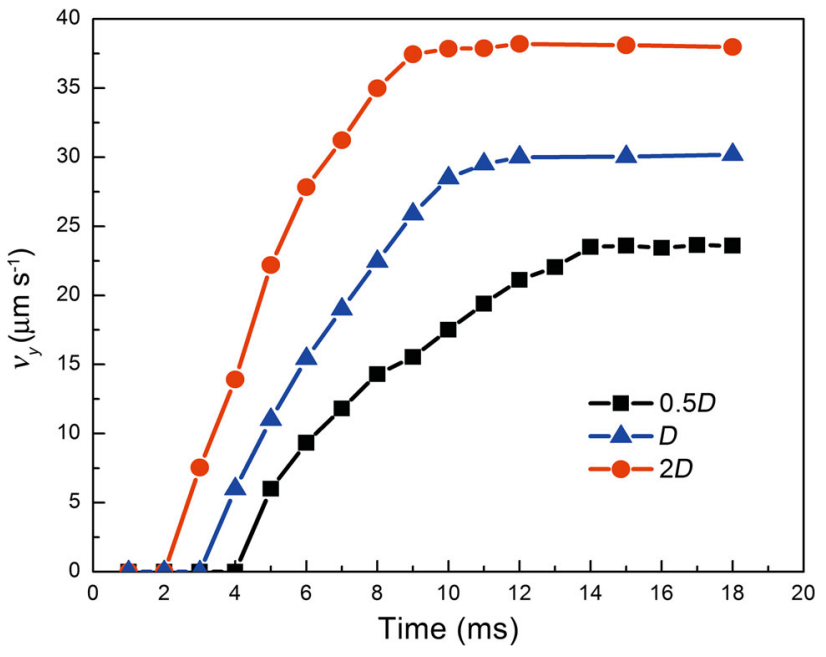

Fig. 11 Evolution of velocity at position D of moving interface $\left(v_{y}\right)$ with varying carbon diffusivity in austenite

Fig. 13. It is evident, as expected, that initial interlamellar spacing of the pearlite phase significantly affects the carbon concentration field in austenite and further the moving rate and morphology of the interface. There are two major observations: firstly, decreasing interlamellar spacing produces relatively flat interface at the steady state, whereas the opposite occurs with increasing interlamellar spacing as shown in Fig. 12. Similar to the treatment proposed by Yang et al. [14], a parabolic curve is also used here as analogy to the migrating interface with a purpose to quantitatively describe the bent degree of the interface, as shown in Fig. 12c, which can be described by $y=p x^{2}$. A larger parabolic coefficient $p$ would indicate a more bent interface. It can be seen that the parabolic coefficient $p$ increases from 5.1 to $13.0 \mu \mathrm{m}^{-1}$ with the interlamellar spacing increasing from 0.2 to $0.8 \mu \mathrm{m}$ as shown in Fig. 13 . Secondly, the growth rate of austenite is inversely related to interlamellar spacing. Decreasing the interlamellar

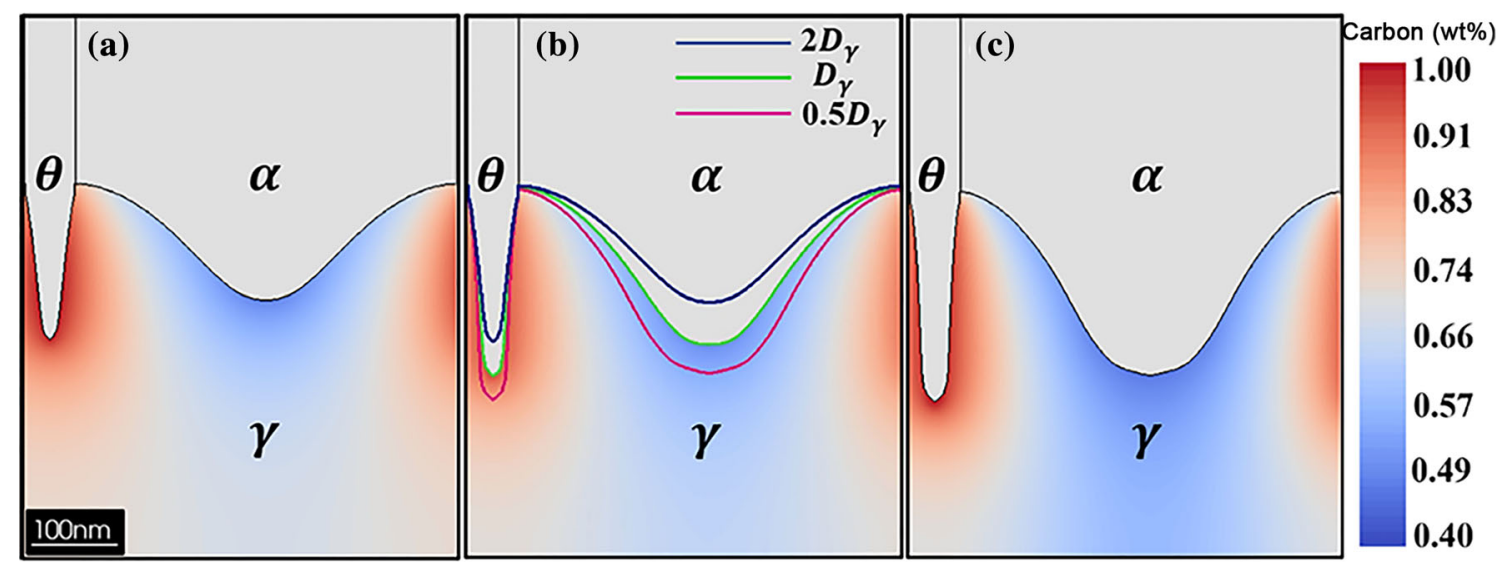

Fig. 10 Interface shapes of growing austenite and carbon concentration profiles at steady state with varied carbon diffusion coefficient: a $2.0 D_{\mathrm{C}}^{\gamma}$; b $D_{\mathrm{C}}^{\gamma} ; \mathbf{c} 0.5 D_{\mathrm{C}}^{\gamma}$ 


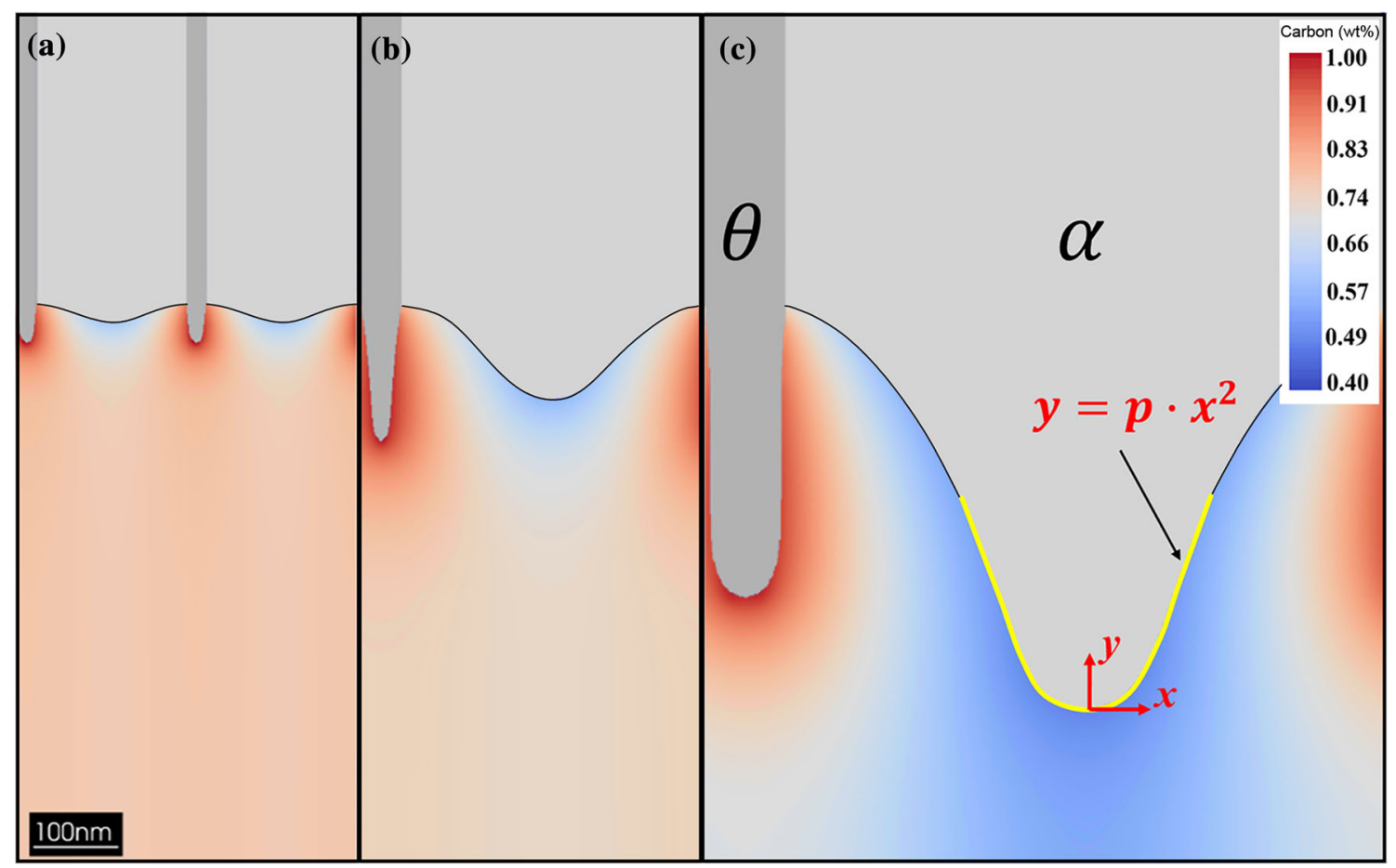

Fig. 12 Interface shapes and carbon concentration profile of growing austenite at steady state with varied interlamellar spacings of $0.2 \mu \mathrm{m}$ a, $0.4 \mu \mathrm{m} \mathrm{b}$ and $0.8 \mu \mathrm{m} \mathbf{c}$

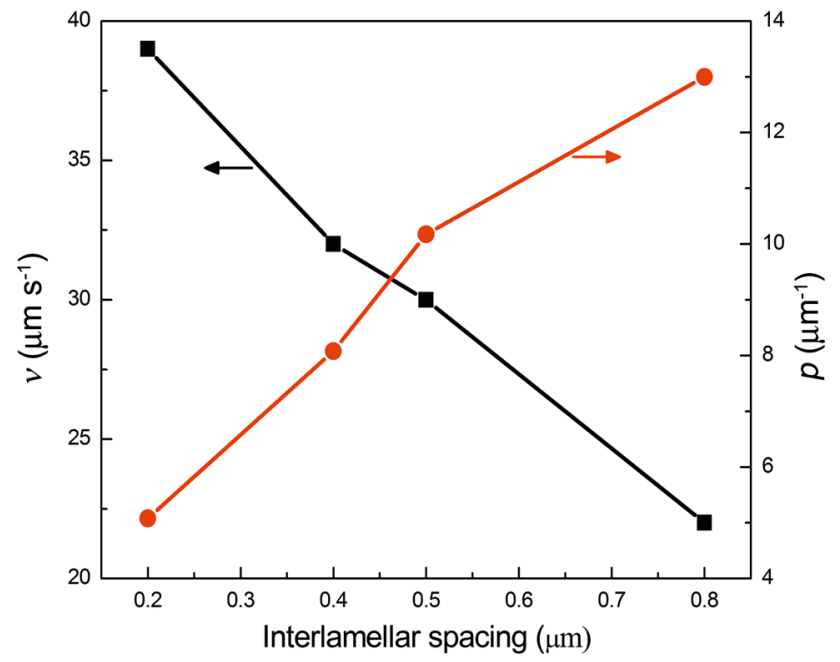

Fig. 13 Growth rate $(v)$ and parabolic coefficient of interface shapes at steady state during austenite formation from pearlite with various interlamellar spacings

spacing from 0.8 to $0.2 \mu \mathrm{m}$ accelerates the interface migration of austenite significantly, as shown in Fig. 13. This can be attributed to the reduction in diffusion distance as the interlamellar spacing decreases. This result is consistent with the experimental findings by Roberts and Mehl [33] and Caballero et al. [34] for C-Mn pearlitic steels. Their observations confirm the acceleration effect of decreased interlamellar spacing on austenite formation. It should be pointed out that the discussions so far apply to only $\mathrm{Fe}-\mathrm{C}$ binary alloys. There are potential limitations without considering the effect of alloying elements, e.g., $\mathrm{Mn}$ and $\mathrm{Cr}$ on the transformation. Schemmann et al. [35] found that the Mn enrichment in cementite would slow down the austenite growth rate during the reaustenitization process. The delaying effect is found even more significant when $\mathrm{Cr}$ is presented [14]. Therefore, further improvement in the current CA model is needed to involve the alloying effect on modeling the interface reactions [36].

\section{Conclusions}

In this work, a modified cellular automaton model is developed to investigate the microstructural behavior of the austenite interface migration during reaustenitization in pearlite steels with lamellar structures. This model can be used to depict the shape of the moving austenite interface, the interface migration rate and the carbon concentration field in austenite under various annealing conditions during the whole austenitization process. The main conclusions are summarized as follows:

1. The CA simulation reveals the evolution of interface migration velocity and shape during austenite growth from initial transient state to a steady state. During initial transient growth of austenite, large moving rate 
of the interface in the region along the $\alpha / \theta$ interface results in a wave-shaped migrating interface.

2. Curvature contribution via the Gibbs-Thomson effect is proved to affect local concentration gradient in front of the austenite interface and further the interface migration behavior. The whole interfaces of austenite migrate with a unity steady-state velocity and remain a fixed shape unless a certain fluctuation of carbon concentration breaks the balance.

3. The carbon diffusivity in austenite and the interlamellar spacing of pearlite strongly affect the interface migration kinematics due to their effect on diffusion rate and diffusion distance, respectively. The simulation results indicate that the interface migration kinematics and morphology of austenite growing within pearlite depend strongly on the carbon diffusivity in austenite and the initial pearlite microstructure. The proposed CA model enables both quantitative and morphological simulation of austenite formation during annealing of pearlite steels.

Acknowledgements This work was financially supported by the National Natural Science Foundation of China (Nos. 51371169 and 51401214).

\section{References}

[1] D.A. Porter, K.E. Easterling, Phase Transformations in Metals and Alloys, 2nd edn. (Chapman \& Hall, London, 1992)

[2] S. Offerman, N. Van Dijk, J. Sietsma, S. Grigull, E. Lauridsen, L. Margulies, H. Poulsen, M.T. Rekveldt, S. Van der Zwaag, Science 298, 1003 (2002)

[3] V. Savran, S. Offerman, J. Sietsma, Metall. Mater. Trans. A 41, $583(2010)$

[4] C.C. Tasan, M. Diehl, D. Yan, M. Bechtold, F. Roters, L. Schemmann, C. Zheng, N. Peranio, D. Ponge, M. Koyama, K. Tsuzaki, D. Raabe, Annu. Rev. Mater. Res. 45, 391 (2015)

[5] A. Ramazani, S. Bruehl, T. Gerber, W. Bleck, U. Prahl, Mater. Des. 57, 479 (2014)

[6] Q. Lai, M. Gouné, A. Perlade, T. Pardoen, P. Jacques, O. Bouaziz, Y. Bréchet, Metall. Mater. Trans. A 47, 3375 (2016)
[7] H. Azizi-Alizamini, M. Militzer, W.J. Poole, Metall. Mater. Trans. A 42, 1544 (2011)

[8] V. Savran, Y. Van Leeuwen, D. Hanlon, C. Kwakernaak, W. Sloof, J. Sietsma, Metall. Mater. Trans. A 38, 946 (2007)

[9] D. Shtansky, K. Nakai, Y. Ohmori, Acta Mater. 47, 2619 (1999)

[10] Y. Li, P. Choi, S. Goto, C. Borchers, D. Raabe, R. Kirchheim, Acta Mater. 60, 4005 (2012)

[11] M. De Graef, M. Kral, M. Hillert, JOM 58, 25 (2006)

[12] F.C. Cerda, I. Sabirov, C. Goulas, J. Sietsma, A. Monsalve, R. Petrov, Mater. Des. 116, 448 (2017)

[13] D. Gaude-Fugarolas, H. Bhadeshia, J. Mater. Sci. 38, 1195 (2003)

[14] Z. Yang, Z. Yang, Y. Xia, C. Zhang, Acta Metall. Sin. 49, 890 (2013). (in Chinese)

[15] Z. Li, Z. Wen, F. Su, R. Zhang, Z. Zhou, J. Alloys Compd. 727, 1050 (2017)

[16] F. Caballero, C. Capdevila, C. García de Andrés, Mater. Sci. Technol. 17, 686 (2001)

[17] K.G.F. Janssens, Computational Materials Engineering an Introduction to Microstructure Evolution (Academic Press, Amsterdam Boston, 2007)

[18] D. Raabe, Annu. Rev. Mater. Res. 32, 53 (2002)

[19] B. Yang, L. Chuzhoy, M. Johnson, Comput. Mater. Sci. 41, 186 (2007)

[20] C. Zheng, D. Raabe, Acta Mater. 61, 5504 (2013)

[21] B. Zhu, Y. Zhang, C. Wang, P.X. Liu, W.K. Liang, J. Li, Metall. Mater. Trans. A 45, 3161 (2014)

[22] C. Halder, L. Madej, M. Pietrzyk, Arch. Civ. Mech. Eng. 14, 96 (2014)

[23] B. Zhu, M. Militzer, Metall. Mater. Trans. A 46, 1073 (2015)

[24] M. Perez, Scr. Mater. 52, 709 (2005)

[25] A. Jacot, M. Rappaz, R. Reed, Acta Mater. 46, 3949 (1998)

[26] B. Su, Z. Han, B. Liu, ISIJ Int. 53, 527 (2013)

[27] K. Kremeyer, J. Comput. Phys. 142, 243 (1998)

[28] M. Enomoto, K. Hayashi, J. Mater. Sci. 50, 6786 (2015)

[29] Y.J. Lan, D.Z. Li, Y.Y. Li, Acta Mater. 52, 1721 (2004)

[30] J.E. Guyer, D. Wheeler, J.A. Warren, Comput. Sci. Eng. 11, 6 (2009)

[31] M. Militzer, H. Azizi-Alizamini, Solid State Phenom. 172, 1050 (2011)

[32] J.O. Andersson, T. Helander, L. Höglund, P. Shi, B. Sundman, Calphad 26, 273 (2002)

[33] G. Roberts, R. Mehl, Trans. Am. Soc. Met. 31, 613 (1943)

[34] F. Caballero, C. Capdevila, C.G. De Andrès, Metall. Mater. Trans. A 32, 1283 (2001)

[35] L. Schemmann, S. Zaefferer, D. Raabe, F. Friedel, D. Mattissen, Acta Mater. 95, 386 (2015)

[36] Y. Xia, M. Enomoto, Z. Yang, Z. Li, C. Zhang, Philos. Mag. 93, 1095 (2013) 